\title{
The attenuation of monochromatic surface waves due to the presence of an inextensible cover
}

\author{
Graig Sutherland*, Trygve Halsne, Jean Rabault, Atle Jensen \\ Department of Mathematics, University of Oslo, Postboks 1053 Blindern, 0316, Oslo, \\ Norway
}

\begin{abstract}
The attenuation of surface gravity waves is an important process associated with air-sea and wave-current interactions. Here we investigate experimentally the attenuation of monochromatic surface gravity waves due to the presence of various surface covers. The surface covers are fixed in space such that they do not advect with the wave motion and are selected such that the bending modulus is negligible for the wave frequencies used in the experiment in order to minimize any flexural effects. Wave attenuation rates are found to be independent of wave steepness and the type of cover used over the tested parameter range. Results are consistent with the theoretical attenuation rate for an inextensible surface cover.
\end{abstract}

Keywords: Surface gravity waves, wave attenuation, surface covers, viscosity

\footnotetext{
*Corresponding author

Email address: graigors@math.uio.no (Graig Sutherland)
} 


\section{Introduction}

Knowledge of the effect of modifying the water surface on attenuating surface waves goes back to antiquity [1]. This changing of the surface rheology can arise from the presence of oil [2], ice [3], or from certain atmospheric and meteorological conditions [4]. Wave attenuation is frequency dependent [5, 6], which generally increases with increasing frequency, although this can depend on the nature of the surface cover [2, 7]. Wave attenuation is an important component of many physical processes such as those associated with air-sea interactions [8], wave-current interactions [9], surface drift [10], and stabilizing wave modulation [11] to name a few.

In the absence of a surface film, wave attenuation is primarily due to the straining motion of the irrotational component of the wave motion (see $[5,6]$ ) and theoretical values have been verified in laboratory experiments in cases where the surface was meticulously cleaned, see $[12,13]$. In the presence of a surface film, a viscous boundary layer develops which acts to increase the attenuation of surface waves (see $[5,6]$ ). If this surface film is elastic then the wave attenuation becomes much more complicated as some of the wave energy can also go into longitudinal waves, also known as Marangoni or dilational waves $[2,14]$.

The inextensible model of [5] has been applied to wave attenuation in ice and require viscosity values several orders of magnitude greater than the molecular value to match observed attenuation rates $[3,15,16,17]$. In ice there are many processes which also attenuate energy such as scattering from ice floes [18] and ice creep [19], which might contribute. These observations are limited to oceanic conditions and often the argument is made that 
the boundary layer is turbulent, predominantly due to the ridges and keels present under the ice, and hence the larger eddy viscosity is justified on these grounds (see [3]).

While laboratory experiments of wave attenuation involving clean water (see [12] and references therein) and water in the presence of surfactants (see [2]) have been performed, there has been little attention paid to investigating inextensible surface covers. More recently, it has been shown by [13] that observations of the attenuation of ocean swell by [20] and [21] are consistent with the theoretical attenuation rate of an inextensible surface cover. However, the physics as to why swell attenuation is best modelled by an inextensible surface cover is not clear.

The main objective of this study is to investigate the attenuation of monochromatic surface waves due to the presence of an inextensible surface cover. The paper is outlined as follows: a brief review of the theoretical background for wave attenuation is described in section 2. A description of the experimental setup is presented in section 3. Section 4 presents the results of the experiment followed a summary and discussion in section 5 .

\section{Theoretical background}

\subsection{Linear wave attenuation}

Wave amplitudes have been observed to attenuate exponentially, i.e.

$$
\frac{\partial a}{\partial x}=-\alpha a
$$

where $a$ is the wave amplitude, $x$ is the distance along the wave flume, and $\alpha$ is the total wave attenuation. Linear wave attenuation arises from viscous 
dissipation within the fluid $[5,6]$ as well as boundary effects from the bottom and sidewalls of a wave flume [22], viscous effects from the air above the interface [23] and the presence of surfactants on the water surface [10, 12, 13].

In the absence of a boundary layer, whether it be at the surface or due to the finite dimensions of a wave flume, the temporal wave attenuation rate, as given by [5], is

$$
\frac{\partial a}{\partial t}=-2 \nu k^{2} a
$$

where $t$ is time, $\nu$ is the kinematic viscosity, and $k$ is the wave number. It is often difficult to clean the surface sufficiently such that there are no particles or natural surfactants present, and thus the clean water attenuation rates are difficult to obtain in wave flumes, see [13].

In the presence of a surface film, the wave attenuation is greater than (2) as the film acts to enhance vorticity in the boundary layer created by the film. In the inextensible limit the boundary condition is assumed to be no-slip and the wave attenuation, as given by [5], is

$$
\frac{\partial a}{\partial t}=-\frac{1}{2} \nu \gamma k a
$$

where $\gamma=\sqrt{\omega / 2 \nu}$ is the inverse boundary layer thickness [5].

The temporal attenuation rate in (2) and (3) can be related to the spatial attenuation rate by using the relation of Gaster [24], i.e.

$$
\frac{\beta}{\alpha}=c_{g}
$$

where $\beta$ is the temporal attenuation rate, as shown in (2) and (3), $\alpha$ is the spatial attenuation rate, and $c_{g}=\partial \omega / \partial k$ is the group velocity. Using (4) with (2) and (3) gives the spatial attenuation rates for clean water,

$$
\alpha_{c l}=2 \nu k^{2} / c_{g}
$$


and

$$
\alpha_{i n}=\frac{1}{2} \nu \gamma k / c_{g}
$$

for the inextensible surface cover.

In general, the ratio $(k / \gamma)$, which is the ratio of the boundary layer thickness to the wavelength, is small with $k / \gamma=2 \times 10^{-3}$ for a $1 \mathrm{~Hz}$ deep water wave and $k / \gamma=2 \times 10^{-2}$ for a $5 \mathrm{~Hz}$ deep water wave. It can easily be shown that $\alpha_{c l} / k=\mathcal{O}(k / \gamma)^{2}$ while $\alpha_{i n} / k=\mathcal{O}(k / \gamma)$, and since $k / \gamma \ll 1$, is consistent with $\alpha_{c l} \ll \alpha_{i n}$.

For insoluble surface covers of finite elasticity and negligible thickness (e.g. a monomolecular surface film) the attenuation rate is given by [10],

$$
\alpha_{e}=\Gamma \alpha_{i n}
$$

where $\Gamma$ is defined as

$$
\Gamma=\left[\frac{2 \mathcal{E}^{2}}{F}+\frac{k}{\gamma} \frac{4(1-\mathcal{E})}{F}\right]
$$

and $\mathcal{E}$ is the non-dimensional elasticity

$$
\mathcal{E}=\frac{E k^{2} \gamma}{\rho \omega^{2}}
$$

${ }_{9} E$ is the film elasticity, $\rho$ is the fluid density and $F=1-2 \mathcal{E}+2 \mathcal{E}^{2}$. The theoretical attenuation rate for an elastic surface cover is shown in Fig. 1. The inextensible limit, i.e. as $E \rightarrow \infty$ and $\alpha_{e} \rightarrow \alpha_{i n}$, and the clean water limit, i.e. $E \rightarrow 0$ and $\alpha_{e} \rightarrow \alpha_{c l}$, are shown. The elastic attenuation rate has a peak value of twice the inextensible limit when $\mathcal{E}=1$. The frequency at which the maximum attenuation occurs is due to a "resonant" type interaction where the dispersion curves of the transverse surface waves and the longitudinal 
waves intersect [2]. This increased attenuation is attributed to a more efficient energy transfer from the transverse waves to the longitudinal waves. Setting $\mathcal{E}=1$ in (9) gives the peak attenuation frequency to be

$$
\omega_{\text {res }}=\left(\frac{2 \nu \rho^{2} g^{4}}{E^{2}}\right)^{\frac{1}{5}} \text {. }
$$

The resonance frequency derived by [2, their equation (6)] differs by a factor of $\rho^{1 / 5}$ from (10), which can be reasoned from dimensional grounds to be a typo in their manuscript.

The difference in deriving (5), (6) and (7) lies in the boundary conditions for the tangential stress $\tau$. Assuming a Hookean tangential stress [10], the stress is written as

$$
\tau=E \Delta x
$$

where $\Delta x$ is the surface strain. In the absence of surface contamination $E=0$ and $\tau=0$. In the inextensible limit $E \rightarrow \infty$, which implies $\Delta x \rightarrow 0$ in order for $\tau$ to be finite. This limit implies that the no-slip condition applies. However, as shown by [25], this is only true in a linear sense and does not apply if nonlinear terms are included.

An additional attenuation process is expected due to the viscous drag of the air above waves. The attenuation of the two fluid system, i.e. air and water, was shown by [23] to be

$$
\alpha_{2 f l}=\alpha_{c l}+\sqrt{2} \frac{\rho_{a}}{\rho}\left(\nu_{a} k^{2}\right)^{1 / 2}(g k)^{1 / 4} / c_{g}
$$

where $\rho_{a}$ and $\nu_{a}$ is the density and kinematic viscosity of the air. The dynamic boundary condition for the two fluid system is continuity in both the tangential and normal stresses at the interface. As shown earlier for deepwater gravity waves, $\alpha_{c l} / k=\mathcal{O}(k / \gamma)^{2}$, while the second term in (12) associated 
with the air drag can be shown to be $\alpha_{a i r} / k \propto\left(\rho_{a} / \rho\right) \sqrt{\nu_{a} / \nu}(k / \gamma)$ where $\alpha_{\text {air }}$ represents the second term in (12). So while the attenuation due to the air viscosity is $\mathcal{O}(k / \gamma)$, identical to boundary layer attenuation, $\rho_{a} \ll \rho$ by three orders of magnitude and will severely limit the effectiveness of the air viscosity to attenuate the waves. Since $k / \gamma$ is also between $10^{-3}$ and $10^{-2}$ the air-side dissipation is expected to be of the same order of magnitude as the water-side.

For laboratory experiments, the finite dimensions of the wave flume will contribute additional wave attenuation [22] with an attenuation rate of

$$
\alpha_{s b}=\nu \gamma k\left(\frac{1}{\sinh 2 k H}+\frac{1}{k B}\right) / c_{g}
$$

where $H$ is the water depth and $B$ is the width of the wave flume. This attenuation is due to the boundary layer dissipation and is $\mathcal{O}(k / \gamma)$ and can assumed to be negligible for large $k H$ for bottom dissipation and for large $k B$ for sidewall attenuation.

\subsection{Linear dispersion relation}

The dispersion relation for the surface transverse wave under a cover of finite thickness $h$ is

$$
\omega^{2}=g k \tanh k H\left(1+\frac{T}{\rho g} k^{2}+\frac{D}{\rho g} k^{4}\right)
$$

where $H$ is the water depth ( $H \rightarrow \infty$ in Fig. 1), $T$ is the surface tension, and $D$ is the bending modulus defined as $D \equiv Y h^{3} / 12\left(1-\mu^{2}\right)$ where $Y$ is the Young's modulus and $\mu$ is the Poisson ratio. The surface tension term is negligible when $T k^{2} /(\rho g) \ll 1$, which occurs for wavelengths $\lambda \gg$ $2 \pi \sqrt{T /(\rho g)}=2 \mathrm{~cm}$. The bending term can be omitted for wavelengths 
$\lambda \gg 2 \pi \sqrt[4]{D /(\rho g)}$, which for our maximum bending modulus $D \approx 2 \times 10^{-5}$ $\mathrm{N} / \mathrm{m}$ (associated with the thick latex cover with $Y=1.5 \mathrm{MPa}, \mu=0.5$ and $h=0.5 \mathrm{~mm})$ gives $\lambda \gg 4 \mathrm{~cm}$. In addition, changes to the dispersion relation (14) due to the attenuation associated with the surface boundary layer are of order $(k / \gamma)$ and is negligible for for the frequencies used in this experiment [10].

\section{Experimental setup}

Experiments were conducted in the $24.6 \mathrm{~m}$ long and $0.5 \mathrm{~m}$ wide wave flume located in the Hydrodynamics Laboratory at the University of Oslo. The wave flume was filled with freshwater to a depth of $0.6 \mathrm{~m}$. Waves were generated with a hydraulic-driven piston. An absorbing beach is located at the opposite end of the wave flume to the wave generator in order to minimize the reflection of wave energy. The reflection coefficient of the absorbing beach was calculated using the WaveAr software [26] to be less than $4 \%$.

Three different surface covers were used in the experiments: a woven polypropylene cover with thickness of $0.3 \mathrm{~mm}$, and two latex covers of thickness 0.25 and $0.5 \mathrm{~mm}$. The latex covers were similar to the ones used by [27], while the woven polypropylene cover is typical to that used in gardens as a weed barrier. Each cover has a width of $0.5 \mathrm{~m}$, which spanned the width of the wave flume, with a length of $17 \mathrm{~m}$ for the polypropylene and $15 \mathrm{~m}$ for the two latex covers. Each surface cover was fixed to the wave flume at 6.4 $\mathrm{m}$ from the wave paddle. The cover was fastened to a horizontal bar placed along the width of the flume and $10 \mathrm{~cm}$ above the still water line to prevent over wash of the waves as they encountered the cover. The covers were not 
fixed at the sidewalls of the wave flume.

For the wave damping experiments, monochromatic waves were used at frequencies between 1.5 to $2.4 \mathrm{~Hz}$ and a wave steepness $a k$ between 0.05 and 0.15. Table 1 shows the experimental parameters used in this study. Wave elevation along the flume was measured using an array of ULS Advanced ultrasonic wave gauges manufactured by Ultralab. Each wave gauge has a resolution of $0.18 \mathrm{~mm}$ and sampled the surface elevation at $250 \mathrm{~Hz}$. The array consisted of 3 wave gauges equally spaced $1.8 \mathrm{~m}$ apart from each other. The array was traversed along the length of the wave flume at discrete time intervals to increase spatial coverage. Since the cover is fixed to the wave flume, there is no advection of the surface cover and the wave field can be assumed to be steady state after some initial set-up time, which we take a cautious value of 5 minutes. After the wave field has clearly achieved a steady state, the wave array is shifted every 3 minutes by $0.7 \mathrm{~m}, 2.7 \mathrm{~m}, 5.4 \mathrm{~m}$, and $8.1 \mathrm{~m}$ relative to the leading edge of the surface cover. The first wave gauge is located at $0.3 \mathrm{~m}$ upstream of the cover, thus elevation measurements are obtained at $-0.3 \mathrm{~m}, 0.4 \mathrm{~m}, 1.5 \mathrm{~m}, 2.2 \mathrm{~m}, 2.4 \mathrm{~m}, 3.3 \mathrm{~m}, 4.0 \mathrm{~m}, 4.2 \mathrm{~m}, 5.1 \mathrm{~m}$, $6.0 \mathrm{~m}, 6.9 \mathrm{~m}, 7.8 \mathrm{~m}, 8.7 \mathrm{~m}, 9.6 \mathrm{~m}$, and $11.4 \mathrm{~m}$ relative to the leading edge of the surface cover. To avoid the possible contamination of reflected waves at the leading edge of the cover only measurements downstream of this edge are used. 


\section{Results}

The wave elevation along the wave flume is calculated using

$$
a(x)=\sqrt{\int_{f_{0}-\Delta f}^{f_{0}+\Delta f} S(f) d f}
$$

where $S(f)$ is the wave spectrum, $f_{0}$ is the forcing frequency and $\Delta f$ the bandwidth of the wave spectrum and is chosen to be $\Delta f=0.1 \mathrm{~Hz}$. Our

results are not sensitive to the choice of $\Delta f$ as long as $\Delta f \ll f_{0}$ so not to include bounded waves. Wave spectra for various locations along the tank can be seen in Fig. 2 for the $2.2 \mathrm{~Hz}$ wave propagating under the thin latex cover. The peak of each wave spectrum is seven orders of magnitude greater than the noise floor outside the spectral bandwidth (shown by the shaded grey region).

The wave amplitude, as a function of distance along the wave flume, is shown in Fig. 3 to attenuate exponentially. The wave amplitude is normalized by the amplitude measured before the surface cover, i.e. $a_{0}=a(x=0.4 \mathrm{~m})$, and the distance is normalized by $k$ calculated by (14). The wave attenuation rate is calculated using a least-squares fit of all the observed values and the error bars show two standard deviations of the best fit parameters. The exponential model for wave attenuation fits the observed values very well and is independent of the wave amplitude for the range of wave steepness used in the experiment.

The observed attenuation rates for all the frequencies and surface covers are shown in Fig. 4. These attenuation rates are compared with the theoretical attenuation rates for the clean and inextensible limits along with that for the sidewalls and bottom. For the theoretical attenuation rates, the 
kinematic viscosity is assumed to be $\nu=1.1 \times 10^{-6} \mathrm{~m}^{2} \mathrm{~s}^{-1}$ and the density is $\rho=1.0 \times 10^{3} \mathrm{kgm}^{-3}$. Results are generally consistent with the inextensible limit of Lamb [5]. The error relative to the inextensible limit for each frequency and surface cover is shown in Figure 5.

\section{Summary and Discussion}

Presented is a series of laboratory experiments using fixed surface covers to valid wave attenuation due to an inextensible cover. The surface cover had to be sufficiently flexible to follow the surface of the propagating waves. Latex, of which two different thicknesses were tested, as well as polypropylene were chosen as they both possessed the surface following condition yet have different elastic properties. For the frequencies used in this experiment, the choice of the surface cover had little effect on the attenuation rate. The stiffer covers, such as the thick latex and the polypropylene, had larger errors associated with the exponential fits, which is presumed to arise from these covers not following the surface as accurately as the thin latex.

For frequencies less than $2 \mathrm{~Hz}$, observations of the attenuation rate were consistent with the inextensible limit of Lamb [5], within two standard deviations of the best fit parameters. However, for frequencies greater than 2 $\mathrm{Hz}$, the attenuation rates exceed the inextensible limit by 20 to $90 \%$. While the presence of longitudinal waves, due to the elasticity of the surface cover, can lead to a peak attenuation rate of twice the inextensible limit, which can be seen in Fig. 1, this seems unlikely as the different cover materials yield similar results. Henderson et al. [13] also observed wave attenuation rates of roughly twice the inextensible value of (3) using sheets of cling wrap for the 
surface cover, which they hypothesized were due to the presence of longitudinal waves. However, this maximum attenuation rate exists for a relatively narrow bandwidth (see [2]) and it is unclear whether this should be applied over the frequency range of $1 \mathrm{~Hz}$ to $4 \mathrm{~Hz}$ used in their experiments [13].

The effect of the added mass of the surface cover on the wave attenuation is expected to be negligible as the relative density of polypropylene and latex to freshwater is 0.92 and 0.96 respectively. The cover will also affect the dispersion relation, but the deviation from (14) will be on the order of $k / \gamma[10]$, which for our frequency range has a maximum value of $8 \times 10^{-3}$ and can safely be ignored.

The elasticity of the surface cover has been ignored in our calculations, but there will exist a certain frequency range where it might be important. In order to estimate the film elasticity we need to linearize the bulk modulus of the sheet. Since the bulk modulus is defined as

$$
Y=\frac{\tau / A}{\Delta x / x_{0}}
$$

where $A$ is the cross-sectional area, $\Delta x$ is the extension and $x_{0}$ is the length, and we want something of the form (11), it is trivial to show that

$$
E=\frac{A Y}{x_{0}} \text {. }
$$

Taking the Young modulus for the thin latex sheet to be $Y=1.0 \times 10^{6} \mathrm{~N} / \mathrm{m}^{2}$, and the dimensions of the sheet to be $b=0.5 \mathrm{~m}, l=15 \mathrm{~m}$ and $h=0.25 \times 10^{-3}$ $\mathrm{m}$ for the width, length, and thickness respectively, we can estimate $E$ from (17) to be about $8 \mathrm{~N} / \mathrm{m}$.

Elastic effects on wave attenuation are tested by a least-squares fit of the observed attenuation rate to (7) and allowing $E$ and $\nu$ to be free parameters. 
The best fit for each cover is shown in Fig. 6. In all cases, the best-fit value for $E$ is greater than $100 \mathrm{~N} / \mathrm{m}$, which has a negligible difference on the attenuation curves over the frequency range used in this experiment. Figure 7 a shows $\mathcal{E}$ as a function of $f$ for different values of $E$ and Fig. $7 \mathrm{~b}$ shows the multiplier $\Gamma$ in $(7)$ as a function of $\mathcal{E}$. For frequencies used in the lab, $E>5 \mathrm{~N} / \mathrm{m}$ leads to values of $\mathcal{E}>10$ (Fig. 7a), which is in the inextensible limit (Fig. 7b). The best-fit values for $\nu$ are larger than the expected value of $\nu=1.1 \times 10^{-6}$, but within two standard deviations $(95 \%$ confidence) with the exception of the polypropylene cover which is three standard deviations larger.

\section{Acknowledgments}

Funding for the experiment was provided by the Norwegian Research Council under the PETROMAKS2 scheme [grant number 233901]. We thank Kai Christensen for the encouragement and many stimulating discussions with regards to the experiments.

\section{References}

[1] J. Scott, The historical development of theories of wave-calming using oil, Hist. Technol. 3 (1978) 163-186.

[2] W. Alpers, H. Hühnerfuss, The damping of ocean waves by surface films: A new look at an old problem, J. Geophys. Res. Oceans 94 (C5) (1989) $6251-6265$.

[3] J. E. Weber, Wave attenuation and wave drift in the marginal ice zone, J. Phys. Oceanogr. 17 (12) (1987) 2351-2361. 
[4] H. Hühnerfuss, W. Walter, G. Kruspe, On the variability of surface tension with mean wind speed, J. Phys. Oceanogr. 7 (4) (1977) 567571.

[5] H. Lamb, Hydrodynamics, 6th Edition, Cambridge university press, 1932.

[6] O. M. Phillips, The Dynamics of the Upper Ocean, Cambridge University Press, 1977, 336 pp.

[7] J. Lucassen, Effect of surface-active material on the damping of gravity waves: A reappraisal, J. Colloid Interface Sci. 85 (1) (1982) 52-58.

[8] B. Jähne, H. Haußecker, Air-water gas exchange, Ann. Rev. Fluid Mech. 30 (1) (1998) 443-468.

[9] M. S. Longuet-Higgins, R. Stewart, Radiation stress and mass transport in gravity waves, with application to surf beats, J. Fluid Mech. 13 (04) (1962) 481-504.

[10] K. H. Christensen, Transient and steady drift currents in waves damped by surfactants, Phys. Fluids 17 (4) (2005) 042102.

[11] H. Segur, D. Henderson, J. Carter, J. Hammack, C.-M. Li, D. Pheiff, K. Socha, Stabilizing the Benjamin-Feir instability, J. Fluid Mech. 539 (2005) 229-271.

[12] J. W. Miles, Surface-wave damping in closed basins, in: Proceedings of the Royal Society of London A: Mathematical, Physical and Engineering Sciences, Vol. 297, The Royal Society, 1967, pp. 459-475. 
[13] D. Henderson, G. K. Rajan, H. Segur, Dissipation of narrow-banded surface water waves, in: Hamiltonian Partial Differential Equations and Applications, Springer, 2015, pp. 163-183.

[14] R. Dorrestein, General linearized theory of the effect of surface films on water ripples, Nederl. Akad. Van Wetenschapen B 54 (1951) 250-272.

[15] A. K. Liu, E. Mollo-Christensen, Wave propagation in a solid ice pack, J. Phys. Oceanogr. 18 (11) (1988) 1702-1712.

[16] P. Wadhams, V. A. Squire, D. J. Goodman, A. M. Cowan, S. C. Moore, The attenuation rates of ocean waves in the marginal ice zone, J. Geophys. Res. Oceans 93 (C6) (1988) 6799-6818.

[17] F. Ardhuin, P. Sutherland, M. Doble, P. Wadhams, Ocean waves across the arctic: attenuation due to dissipation dominates over scattering for periods longer than 19 s, Geophys. Res. Lett. 43. doi:10.1002/2016GL068204.

[18] A. L. Kohout, M. H. Meylan, An elastic plate model for wave attenuation and ice floe breaking in the marginal ice zone, J. Geophys. Res. 113. doi:10.1029/2007JC004434.

[19] P. Wadhams, Attenuation of swell by sea ice, J. Geophys. Res. 78 (18) (1973) 3552-3563.

[20] F. E. Snodgrass, G. W. Groves, K. Hasselmann, G. Miller, W. Munk, W. Powers, Propagation of ocean swell across the pacific, Proc. R. Soc. A 259 (1103) (1966) 431-497. 
[21] F. Collard, F. Ardhuin, B. Chapron, Monitoring and analysis of ocean swell fields from space: New methods for routine observations, J. Geophys. Res. Oceans 114 (C7).

[22] W. Van Dorn, Boundary dissipation of oscillatory waves, J. Fluid Mech. 24 (04) (1966) 769-779.

[23] B. Dore, Some effects of the air-water interface on gravity waves, Geophys. Astrophys. Fluid Dyn. 10 (1) (1978) 215-230.

[24] M. Gaster, A note on the relation between temporally-increasing and spatially-increasing disturbances in hydrodynamic stability, J. Fluid Mech. 14 (1962) 222-224.

[25] D. M. Henderson, H. Segur, The role of dissipation in the evolution of ocean swell, J. Geophys. Res. Oceans 118 (10) (2013) 5074-5091.

[26] B. J. Landry, M. J. Hancock, C. C. Mei, M. H. García, WaveAR: A software tool for calculating parameters for water waves with incident and reflected components, Computers \& Geosciences 46 (2012) 38-43.

[27] L. Deike, J.-C. Bacri, E. Falcon, Nonlinear waves on the surface of a fluid covered by an elastic sheet, J. Fluid Mech. 733 (2013) 394-413. 
Table 1: Experimental parameters. Thin refers to the thin latex, thick is the thick latex, and poly is the polypropylene cover.

\begin{tabular}{ccccc} 
cover & $f / \mathrm{Hz}$ & $\lambda / \mathrm{m}$ & $a_{0} k$ & $\alpha / k \times 10^{-3}$ \\
\hline thin & 1.00 & 1.55 & 0.04 & $2.11 \pm 1.04$ \\
& 1.50 & 0.70 & 0.07 & $3.41 \pm 0.26$ \\
& 1.75 & 0.51 & 0.09 & $3.12 \pm 1.36$ \\
& 2.20 & 0.32 & 0.10 & $5.48 \pm 0.54$ \\
& 2.40 & 0.27 & 0.12 & $6.36 \pm 0.68$ \\
thick & 1.00 & 1.55 & 0.04 & $2.52 \pm 0.82$ \\
& 2.00 & 0.39 & 0.09 & $5.61 \pm 1.20$ \\
& 2.20 & 0.32 & 0.14 & $6.45 \pm 1.64$ \\
& 2.40 & 0.27 & 0.11 & $9.31 \pm 5.44$ \\
poly & 1.50 & 0.70 & 0.07 & $3.16 \pm 0.24$ \\
& 2.00 & 0.39 & 0.09 & $4.81 \pm 0.14$ \\
& 2.20 & 0.32 & 0.09 & $5.61 \pm 1.20$ \\
& 2.40 & 0.27 & 0.12 & $8.24 \pm 1.82$ \\
\hline
\end{tabular}




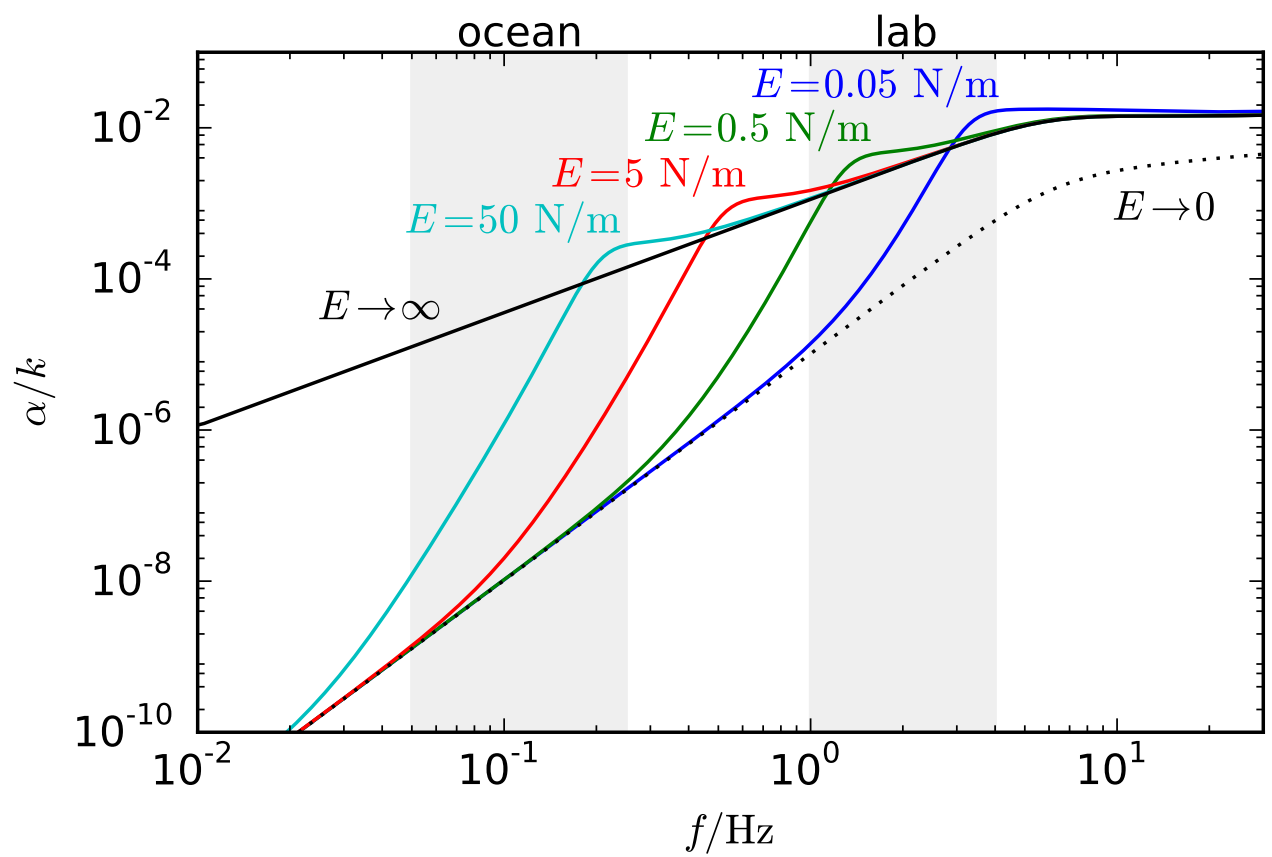

Figure 1: Attenuation rate normalized by the wave number $k$ as a function of frequency. The grey shaded region on the left shows a typical spectral range for ocean waves of 0.05 $\mathrm{Hz}$ to $0.25 \mathrm{~Hz}$ and the region on the right shows a typical range for laboratory waves of $1 \mathrm{~Hz}$ to $4 \mathrm{~Hz}$. The inextensible $(E \rightarrow \infty)$ and clean $(E \rightarrow 0)$ limits are shown along with the attenuation rate for different film elasticity. 

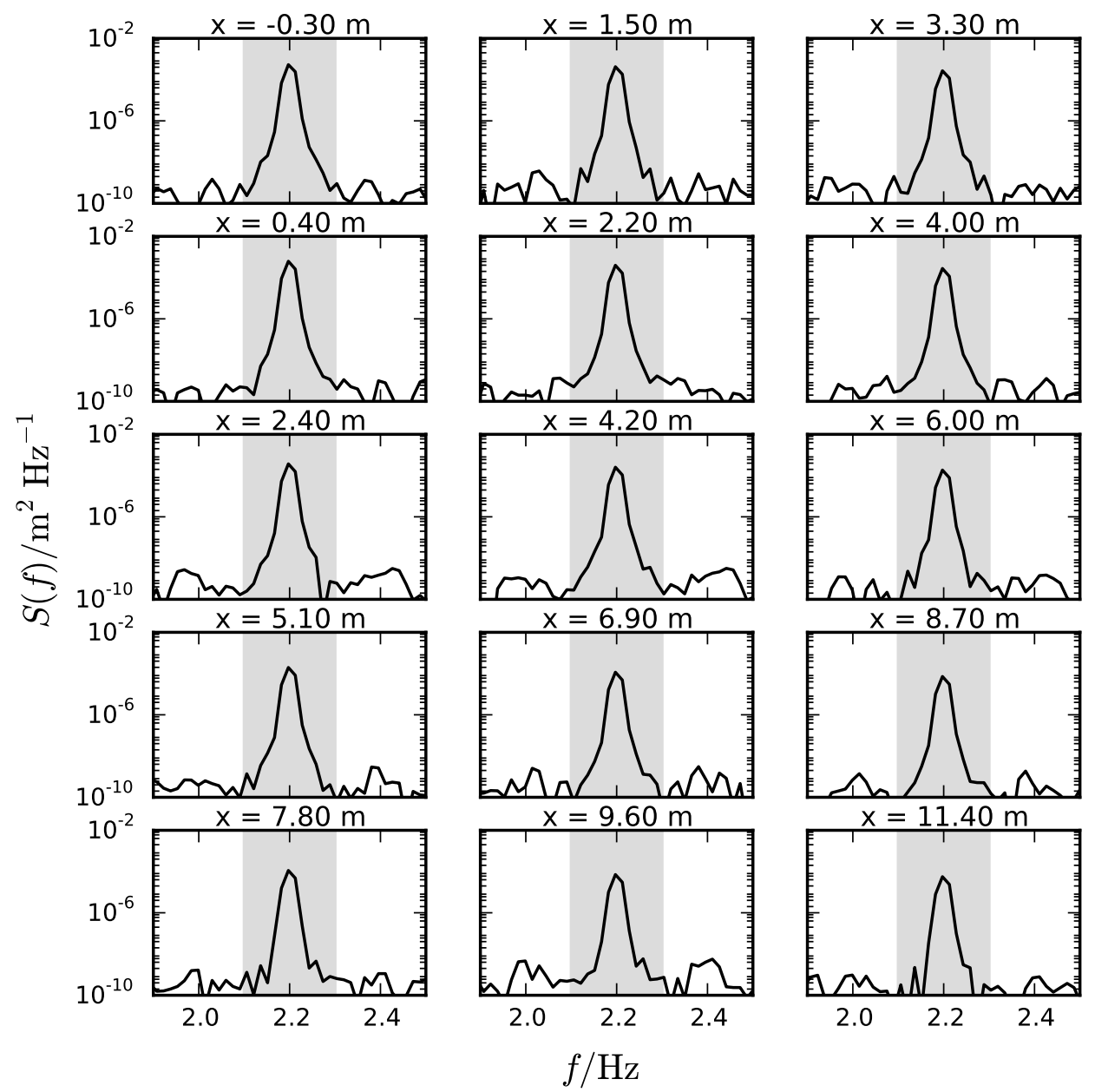

Figure 2: Wave spectra calculated at 15 different locations for the $2.2 \mathrm{~Hz}$ wave with a steepness of $a_{0} k=0.10$ propagating under the thin latex cover. The grey shaded region denotes the spectral bandwidth used to obtain the amplitude. 


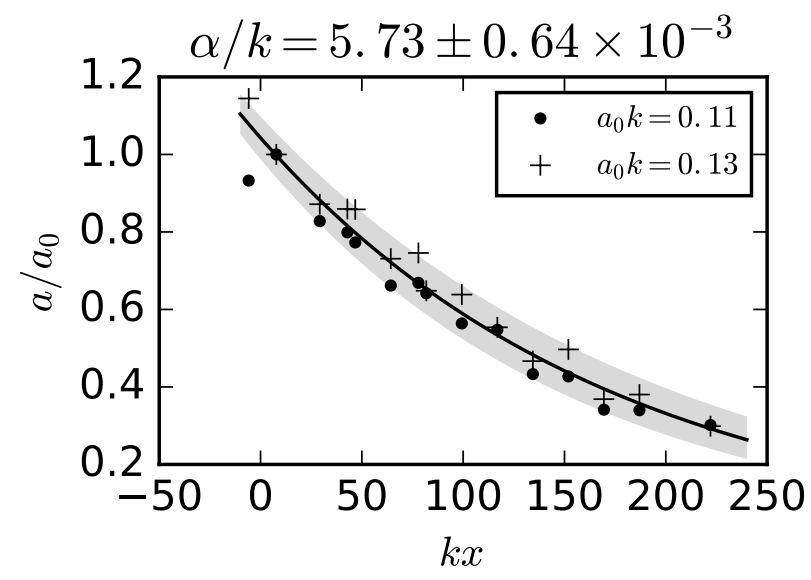

Figure 3: Wave attenuation with the thin latex cover for a $2.20 \mathrm{~Hz}$ wave for two different wave steepness. The attenuation rate does not vary for the two different wave steepness. The grey shaded region shows two standard deviations of the least-squares fit. 


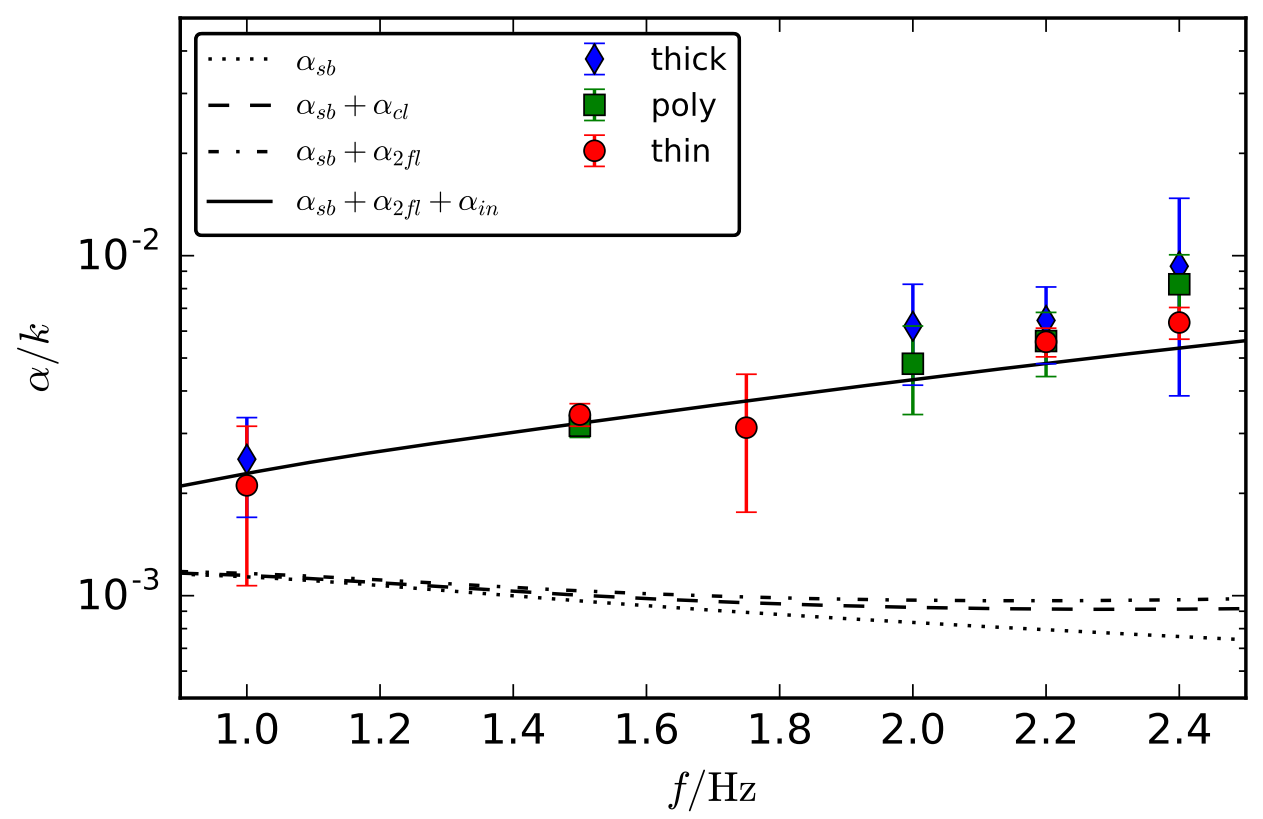

Figure 4: Wave attenuation rate normalized by the wavenumber $k$ as a function of frequency for each surface cover (thick $=0.5 \mathrm{~mm}$ latex, poly $=0.3 \mathrm{~mm}$ polypropylene, thin $=0.25 \mathrm{~mm}$ latex). The error bars show two standard deviations of the least-squares fit to the attenuation curve. 


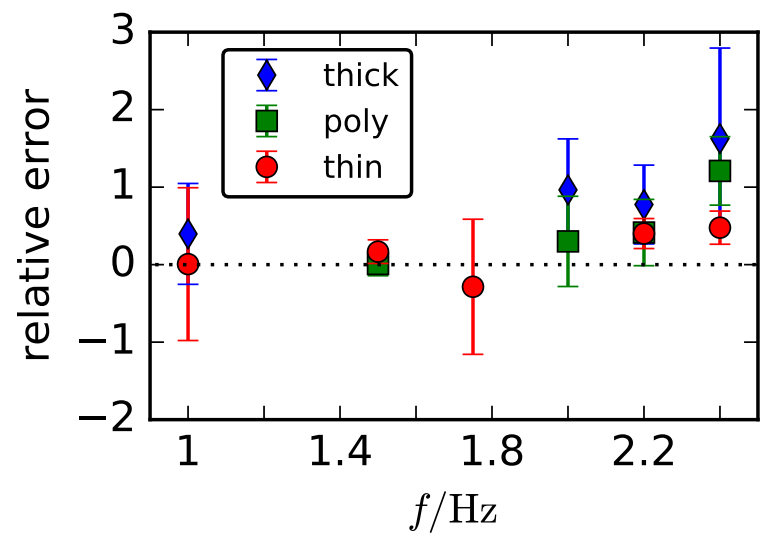

Figure 5: Relative error of the observed attenuation rate with the theoretical value $\left(\alpha_{i n}+\right.$ $\left.\alpha_{s b}\right)$ as a function of frequency for the various surface covers. The error bars show two standard deviations of the least-squares fit to the attenuation curve. 


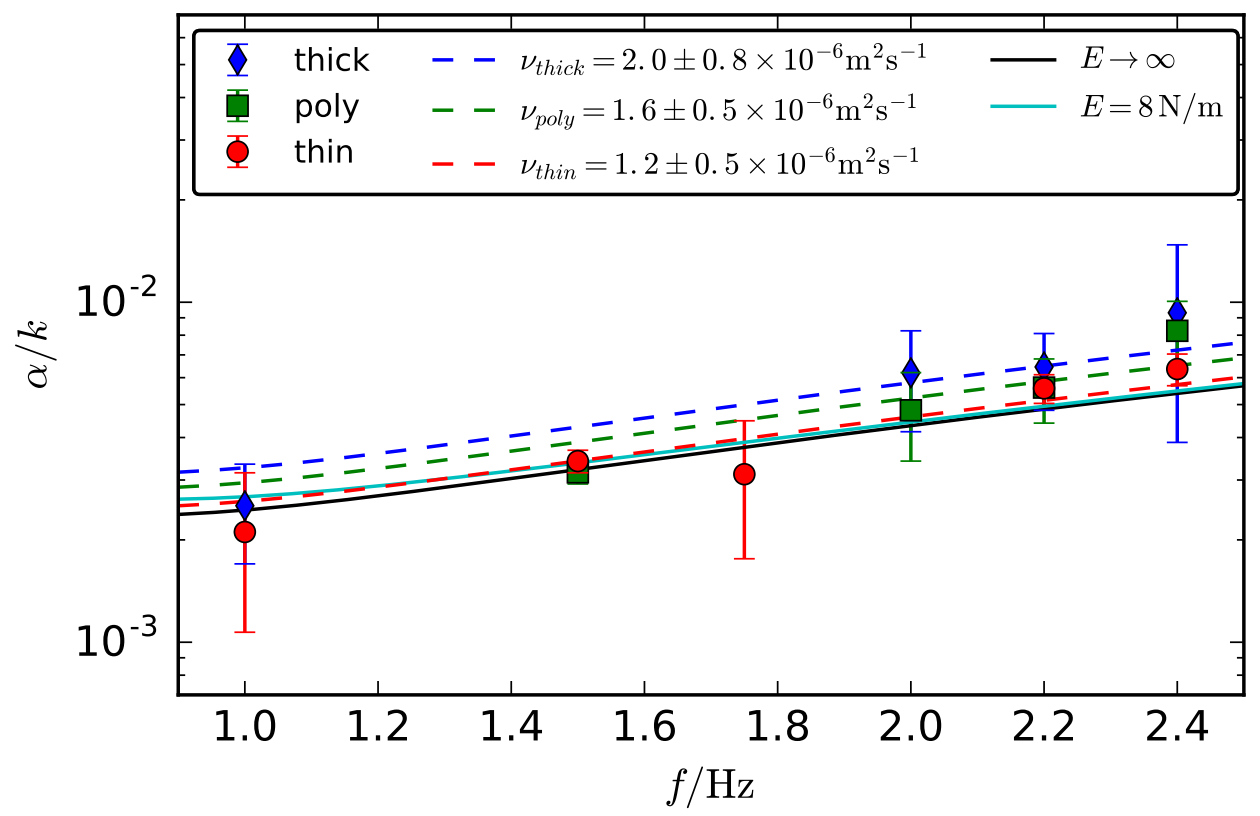

Figure 6: Wave attenuation rate normalized by the wavenumber $k$ as a function of frequency for each surface cover (thick $=0.5 \mathrm{~mm}$ latex, poly $=0.3 \mathrm{~mm}$ polypropylene, thin $=$ $0.25 \mathrm{~mm}$ latex). The black line shows the theoretical value for $\nu=1.1 \times 10^{-6} \mathrm{~m}^{2} \mathrm{~s}-1$. The film elasticity and viscosity are used as free parameters and fit to the observed attenuation rates. The error bars show two standard deviations of each individual attenuation rate. 

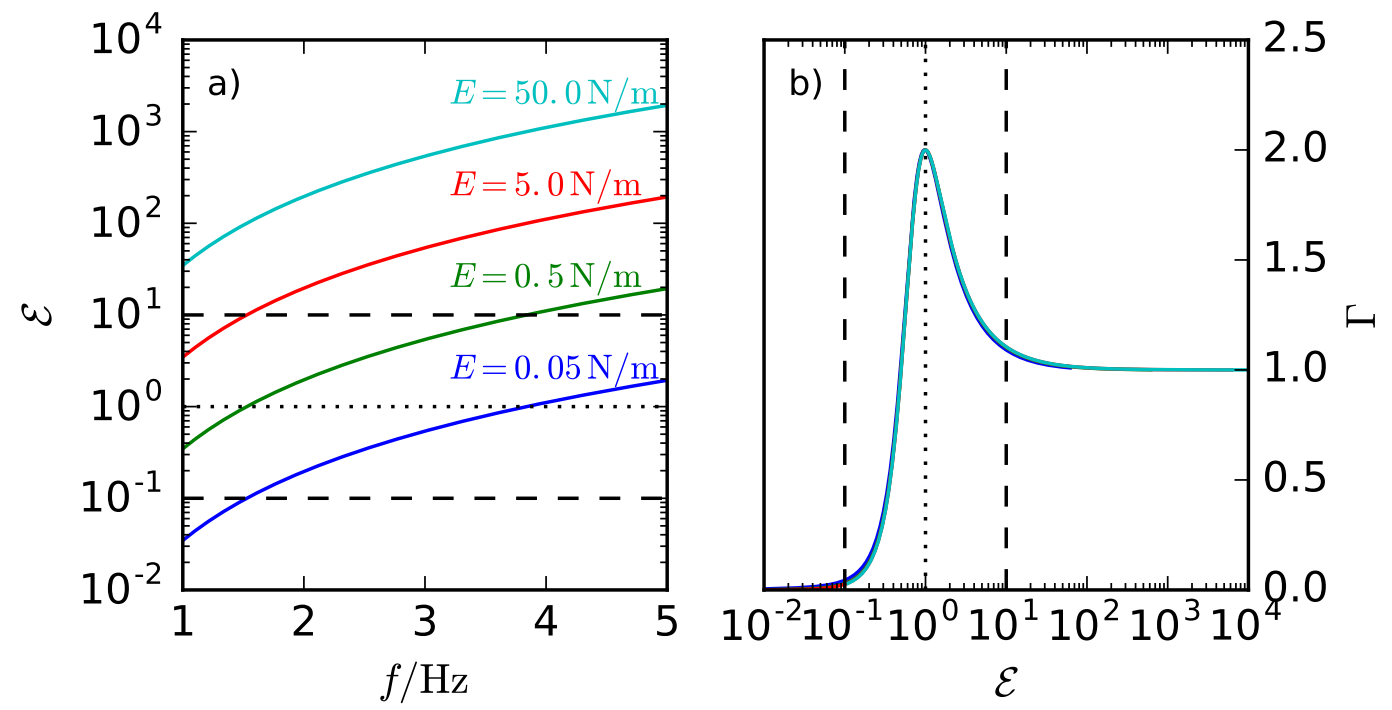

Figure 7: a) Non-dimensional elasticity $\mathcal{E}$ as a function of frequency for different values for the film elasticity. Attenuation rates are twice the inextensible limit when $\mathcal{E}=1$, which is shown by the blacked dotted line. b) The attenuation multiplication factor $\Gamma$ for finite film elasticities as a function of $\mathcal{E}$ showing the transition from the clean surface to an inextensible surface film. The black dotted line shows $\mathcal{E}=1$ and the dashed line shows $\mathcal{E}=10^{-1}$ and $\mathcal{E}=10$, which corresponds to the clean and inextensible limits respectively. 